\title{
AVALIAÇÃO DE METODOLOGIA ELETROQUíMICA NO MONITORAMENTO DA CONVERSÃO DE ÓLEO DE GIRASSOL EM BIODIESEL
}

\author{
Rogério Adelino de Sousa e Caue Ribeiro* \\ Embrapa Instrumentação, R. Quinze de Novembro, 1452, 13560-970 São Carlos - SP, Brasil
}

Recebido em 11/1/11; aceito em 24/5/11; publicado na web em 26/7/11

\begin{abstract}
STUDY OF AN ELECTROCHEMICAL METHOD TO SUNFLOWER OIL TO BIODIESEL CONVERSION ASSESSMENT. The development of analytical procedures to evaluate transesterification process is still a challenge in biodiesel production. Then, this paper shows an electroanalytical methodology to transesterification process assessment, proposing the application of nanostructured $\mathrm{TiO}_{2}$ electrodes. The results showed, for sunflower oil - methanol reaction catalyzed by $\mathrm{KOH}$, a reduction peak in $-1050 \mathrm{mV}$ and the gradual appearance of a second peak at $-1160 \mathrm{mV}$. This peak was observed as originated by the transesterification process and is probably related to intermediates. By measuring the intensity of this peak a kinetic profile was determined, showing that the conversion is almost finished in $2 \mathrm{~h}$.
\end{abstract}

Keywords: biodiesel; electroanalytical measurement; nanostructured $\mathrm{TiO}_{2}$.

\section{INTRODUÇÃO}

Os altos preços do petróleo e a necessidade de fontes energéticas que reduzam a emissão de gases do efeito estufa, responsáveis pelo aquecimento global, impulsionam o desenvolvimento e a utilização de combustíveis renováveis, os quais não interferem no ciclo do carbono. Uma alternativa à gasolina é o etanol, no qual os Estados Unidos são hoje líder na produção, porém o Brasil é vice-líder mundial e detém grandes avanços tecnológicos na sua produção. Analogamente ao etanol, o biodiesel é a alternativa renovável mais usada no Brasil para o diesel de petróleo. Contudo, a pesquisa e o desenvolvimento do biodiesel estão longe do patamar tecnológico obtido no etanol. ${ }^{1,2}$

Tecnicamente, o biodiesel é uma mistura de ésteres provenientes da reação de óleos vegetais ou gorduras animais (constituídos basicamente por três cadeias de ácidos graxos ligados a uma molécula de glicerina, chamados de triglicerídeos ou triacilglicerídeos (TAG) $)^{3}$ com metanol ou etanol catalisados por $\mathrm{NaOH}, \mathrm{KOH}, \mathrm{H}_{2} \mathrm{SO}_{4}$ ou enzimas, por exemplo. ${ }^{4,5}$ Os catalisadores básicos tradicionais são largamente utilizados na indústria para obtenção de biodiesel, pois, além de serem menos agressivos aos equipamentos, apresentam atividades até 4.000 vezes superiores às verificadas para os ácidos minerais. ${ }^{6,7}$ Essa reação é conhecida como transesterificação dos triglicerídeos ${ }^{8}$ e está representada pela Equação 1.
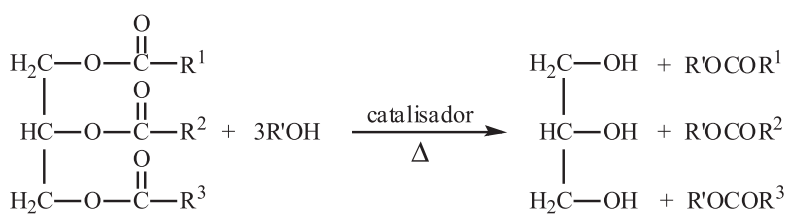

Os $\mathrm{R}^{1}, \mathrm{R}^{2}$ e $\mathrm{R}^{3}$ representam cadeias carbônicas (iguais ou não) e $\mathrm{R}^{\prime} \mathrm{OH}$ representa o álcool cogerador.

Um dos principais desafios na aplicação industrial da transesterificação é a determinação da taxa de conversão de óleos ou gorduras para ésteres (biodiesel). ${ }^{9}$ Assim, muitos trabalhos de pesquisa têm

*e-mail: caue@cnpdia.embrapa.br proposto diferentes técnicas analíticas com foco em maior velocidade de medida ou acurácia. As técnicas eletroquímicas, como as voltametrias (voltametria cíclica, voltametria linear), a espectroscopia de impedância eletroquímica e a condutometria, podem ser ferramentas poderosas para a determinação de parâmetros físico-químicos e cinéticos da formação do biodiesel. Entretanto, há um obstáculo a ser superado nesta proposta: tanto os TAG quanto os ésteres formados são completamente insolúveis em meio aquoso, mais adequado a processos eletroquímicos. Tal obstáculo pode ser superado com o uso de surfactantes ou meio micelar, ${ }^{10,11}$ ou ainda microemulsões. ${ }^{12}$ Fulian et al..$^{13}$ depositaram gotas de $\mathrm{N}, \mathrm{N}, \mathrm{N} \phi, \mathrm{N} \phi$-tetra-hexil-parafenilenediamina (THPD), material oleoso, sobre a superfície de eletrodos de carbono pirolítico e estudaram a eletroatividade desse composto, obtendo resultados satisfatórios.

Assim, este artigo descreve uma metodologia eletroquímica para monitoramento da conversão de um óleo vegetal em biodiesel, utilizando-se um eletrodo de filme nanoestruturado de $\mathrm{TiO}_{2}$ obtido pelo método dos precursores poliméricos. Estes resultados demonstram potencial para desenvolvimento de uma metodologia rápida de determinação da conversão, facilitando o monitoramento deste processo.

\section{PARTE EXPERIMENTAL}

Os reagentes usados foram de grau analítico, PA, ou de grau cromatográfico, dependendo da aplicação desejada. Nos dois casos os reagentes foram usados sem purificação prévia. Todas as soluções foram preparadas com água purificada em um sistema Millipore Milli-Q (resistividade $<18 \mathrm{M} \Omega \mathrm{cm}$ ).

Foi utilizado óleo de girassol comercial como substrato para as transesterificações e para os experimentos eletroquímicos e dimetilssulfato de sódio (DSS) para a formação das emulsões. As proporções usadas foram $50,0 \mathrm{~mL}$ de metanol para $50,0 \mathrm{~mL}$ de óleo de girassol $\left(0,0481 \mathrm{~mol} \text { de óleo, baseados na massa molar de 895,9 } \mathrm{g} \mathrm{mol}^{-1}\right)^{14}$ e 1,0 g de DSS, formando uma emulsão. Como catalisador para a reação utilizou-se $\mathrm{KOH}$ (0,5\% m:m do óleo) e temperatura constante de $70{ }^{\circ} \mathrm{C}$. Esta metodologia foi usada em todos os experimentos e, para confirmação dos perfis eletroquímicos obtidos, repetiram-se o procedimento e as medidas utilizando-se óleo de soja e de canola, também comerciais. Para confirmação da obtenção de biodiesel ao 
final do processo, uma amostra de óleo de girassol e do produto final da reação, devidamente decantado para separação da fração glicerina, foram qualitativamente analisadas separadamente, por meio de ressonância magnética nuclear, em um espectrômetro de ressonância magnética nuclear marca Varian, modelo Inova 400, com campo de 9,4 T (equivalente a $400 \mathrm{MHz}$ para ${ }^{1} \mathrm{H}$ ), em 32 scans, para melhor relação sinal/ruído. O pulso utilizado foi de $10 \mu$ s, com tempo de aquisição de $5 \mathrm{~s}$ e intervalo entre pulsos de $10 \mathrm{~s}$. Uma alíquota de $10 \mu \mathrm{L}$ de amostra, óleo de girassol ou do produto final da reação, foi dissolvida em $500 \mu \mathrm{L}$ de clorofórmio deuterado com tetrametilsilano como padrão interno.

Para os estudos eletroquímicos, foi usado um potenciostato/galvanostato Autolab gerenciado pelo programa GPES 4.9 (Eco Chemie). A célula eletroquímica foi formada por três eletrodos, sendo o eletrodo de referência e o contraeletrodo um fio de platina de $1,0 \mathrm{~cm}$ de comprimento. O eletrodo de trabalho de dióxido de titânio foi construído seguindo o método dos precursores poliméricos, seguindo o método descrito por Malagutti et al.: ${ }^{15}$ foi depositada uma camada da resina sobre uma superfície de Ti metálico polido (substrato condutor) por spin coating, com rotação inicial de $1000 \mathrm{rpm}\left(15 \mathrm{rpm} \mathrm{s}^{-2}\right)$ por $10 \mathrm{~s}$ e uma segunda velocidade de rotação de $5000 \mathrm{rpm}$ durante $40 \mathrm{~s}$. Os filmes foram tratados a $300^{\circ} \mathrm{C}$, por $2 \mathrm{~h}$, em forno elétrico convencional para pirólise da resina e cristalizados a $450{ }^{\circ} \mathrm{C}$, por $2 \mathrm{~h}$, para formar o filme nanoestruturado de $\mathrm{TiO}_{2}$ anatase. $\mathrm{O}$ procedimento foi repetido quatro vezes, para formação de um filme espesso.

\section{Célula eletroquímica}

Para a realização dos experimentos eletroquímicos foi construída uma célula eletroquímica completa, composta de uma cuba de vidro encamisada para o controle de temperatura, tampa construída em Teflon ${ }^{\circledR}$ com $o$-ring para controle atmosférico, eletrodo de referência e contraeletrodo, ambos de fio de platina, e um suporte construído de Teflon ${ }^{\circledR}$ para o acondicionamento do eletrodo de trabalho.

\section{Determinação da superfície eletroativa do eletrodo de trabalho}

A área da superfície eletroativa do eletrodo de filme nanoestruturado de $\mathrm{TiO}_{2}$ foi determinada pelo método das velocidades de varredura, uma solução $5,00 \mathrm{mmol} \mathrm{L}^{-1}$ de $\mathrm{K}_{3} \mathrm{Fe}(\mathrm{CN})_{6}$, em $\mathrm{KCl}$ $0,5 \mathrm{~mol} \mathrm{~L}^{-1}$, foi usada como sonda eletroquímica. Foram obtidas as correntes de pico anódico $\left(I_{p, \mathrm{a}}\right)$ e catódico $\left(I_{p, \mathrm{c}}\right)$ em diferentes velocidades de varredura $(v)\left(10,25,35,50,69,75\right.$ e $\left.100 \mathrm{mV} \mathrm{s}^{-1}\right) .{ }^{16}$ Com o auxílio da Equação de Randles e Sevcik (Equação 2), calculou-se a área eletroativa do eletrodo de filme nanoestruturado de $\mathrm{TiO}_{2}$ para cada velocidade de varredura usando as $I_{p, \mathrm{a}}$ do $\mathrm{K}_{3} \mathrm{Fe}(\mathrm{CN})_{6}$. O valor da área eletroativa foi calculado através da média aritmética dos valores das áreas obtidas em cada velocidade de varredura estudada, sendo de $2,7 \times 10^{-4} \pm 0,13 \times 10^{-5} \mathrm{~cm}^{2}$.

$$
I_{p, \mathrm{a}}=2,69 \mathrm{x} 10^{5} \cdot \mathrm{n}^{3 / 2} \cdot A \cdot D \mathrm{o}^{1 / 2} \cdot[\mathrm{Ox}] \cdot v^{1 / 2}
$$

onde: $I_{p, \mathrm{a}}=$ corrente anódica/A; $\mathrm{n}=$ número de elétrons envolvidos $=1$; $A=$ área eletroativa $/ \mathrm{cm}^{2} ; D o=$ coeficiente de difusão do $\left[\mathrm{K}_{3} \mathrm{Fe}(\mathrm{CN})_{6}\right]$ $=7,70 \times 10^{-6} \mathrm{~cm}^{2} \mathrm{~s}^{-1} ;[\mathrm{Ox}]=\left[\mathrm{K}_{3} \mathrm{Fe}(\mathrm{CN})_{6}\right]_{\text {célula }}=5,00 \mathrm{mmol} \mathrm{L}^{-1}$ e $\mathrm{v}=$ velocidade de varredura/ $/ \mathrm{V} \mathrm{s}^{-1}$.

Após a confecção e a determinação da área eletroativa dos eletrodos de trabalho, foi investigado o comportamento eletroquímico da transesterificação usando voltametria cíclica $(\mathrm{CV})$ nas seguintes condições: velocidade de varredura $(v)$ de $100 \mathrm{mV} \mathrm{s}^{-1}$; intervalo de potencial de -1300 a $1300 \mathrm{mV}$, para ter a maior janela possível para as determinações dos processos; número de ciclos de 46 a 50, resultando em um tempo na ordem de $2 \mathrm{~h}$ para certificação de que a transesterificação se efetuasse, formando a máxima quantidade de biodiesel.

\section{RESULTADOS E DISCUSSÃO}

Foram testados inicialmente vários eletrodos convencionais, como o eletrodo de pasta de carbono, de compósito grafite-poliuretana (GPU) e de filme de $\mathrm{TiO}_{2}$ nanoestruturado sobre Ti metálico. Os experimentos iniciais indicaram que apenas o eletrodo de $\mathrm{TiO}_{2}$ apresentou sinal mensurável durante um experimento teste de transesterificação, o que determinou que todas as avaliações subsequentes fossem feitas com este eletrodo. De fato, deve-se supor alguma interação específica entre os produtos de reação e o eletrodo em si, que permita tal sinal eletroquímico, evento este que está ainda em estudo para melhor compreensão.

A Figura 1 apresenta os registros voltamétricos do branco e da emulsão e o resultado da subtração da curva obtida dos registros da CV da emulsão dos registros em branco, onde o branco foi uma mistura de DSS e metanol nas proporções utilizadas na reação. Notou-se claramente dois processos de redução, o primeiro em -1050 e segundo em -550 mV. Para a confirmação de que os processos eletroquímicos estariam ligados à transesterificação em si, realizou-se um experimento em potencial de redução constante, onde a corrente foi monitorada em função do tempo de reação, como visto na Figura 2. Porém, apenas para o potencial de $-1050 \mathrm{mV}$ houve resultados significativos.

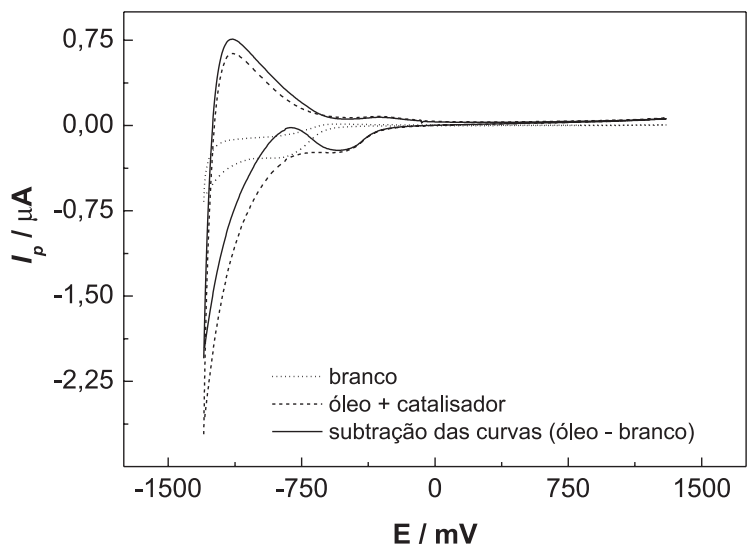

Figura 1. Registros das voltametrias cíclicas do branco, da emulsão e da subtração do gráfico da emulsão do gráfico do branco. O intervalo de potencial usado foi de -1300 a $1300 \mathrm{mV}$

A Figura 2 apresenta duas quedas de corrente significativas, a primeira em torno de $8 \mathrm{~s}$ e a segunda em $213 \mathrm{~s}$. Entretanto, ao se tentar repetir os experimentos, tanto na $\mathrm{CV}$ quanto na variação de $\mathrm{I}_{\mathrm{P}}$ em função do tempo, os resultados não se reproduziram devido ao tempo de registro ter sido insuficiente para que a reação se completasse. Portanto, a transesterificação foi estudada usando o eletrodo nanoestruturado durante $2 \mathrm{~h}$ de reação. Foram registrados 46 ciclos ( $\approx 2$ h de reação) nas condições voltamétricas descritas acima. Como o catalisador para a reação foi o $\mathrm{KOH}$, o primeiro registro obtido foi de uma solução aquosa de $\mathrm{KOH}(0,5 \% \mathrm{~m}: \mathrm{m})$, para conferir se havia alguma resposta eletroquímica frente à presença de $\mathrm{KOH}$. Também se registraram os voltamogramas do metanol puro para investigar a presença de algum sinal, o qual poderia ser um interferente em potencial; registraram-se os voltamogramas do metóxido de potássio (solução metanol KOH 0,5\% m:m) para verificar se havia alguma resposta eletroquímica e, finalmente, registraram-se os voltamogramas referentes à emulsão de metanol, óleo e DSS para identificar algum sinal que poderia ser interferente. A Figura 3 apresenta todos os registros voltamétricos para os experimentos citados acima. 


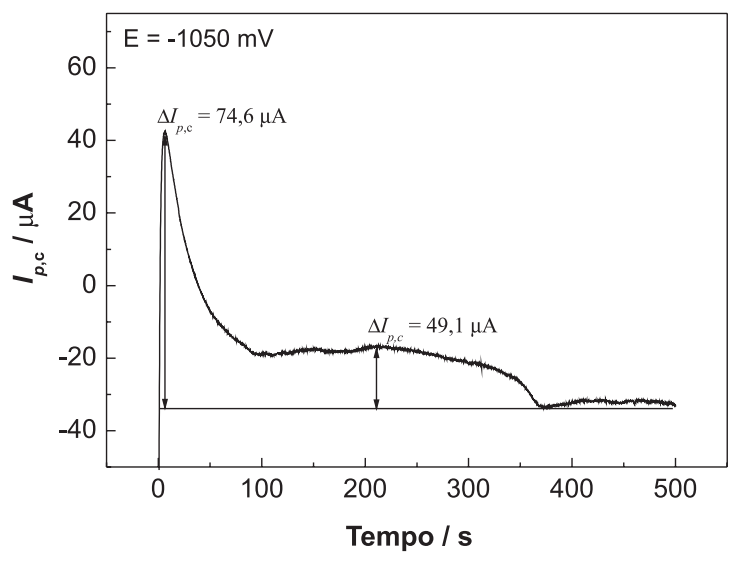

Figura 2. Variação da corrente de pico em função do tempo em potencial constante de $-1050 \mathrm{mV}$

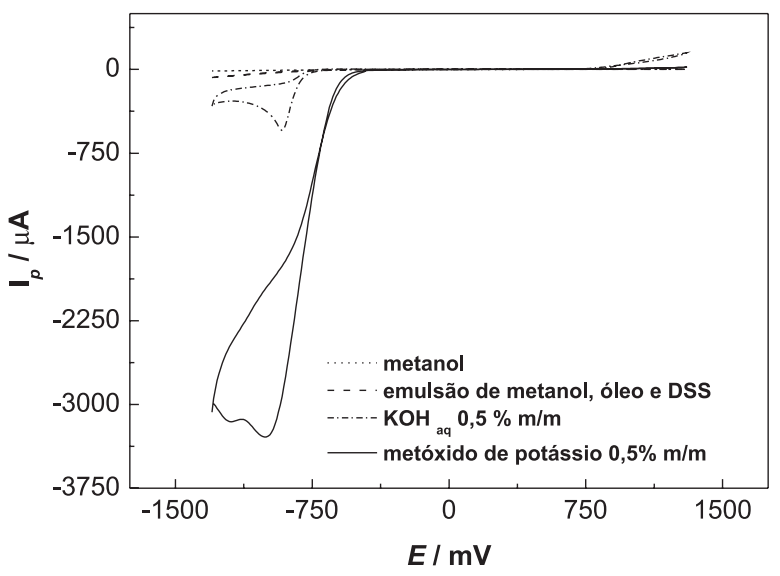

Figura 3. Registros voltamétricos obtidos para a solução aquosa de $\mathrm{KOH}$ $(0,5 \% \mathrm{~m} / \mathrm{m})$; metanol puro; metóxido de potássio $(\mathrm{KOH} \mathrm{0,5 \%} \mathrm{m} / \mathrm{m})$ e emulsão de metanol, óleo e DSS

Baseando-se nos registros voltamétricos apresentados na Figura 3 , identificou-se uma resposta eletroquímica, tanto na solução aquosa quanto na solução metílica de $\mathrm{KOH}$ (metóxido), ou seja, o eletrodo é sensível ao $\mathrm{KOH}$ dissolvido tanto em água, quanto em metanol. $\mathrm{Na}$ solução aquosa de $\mathrm{KOH}$ há um processo claro de redução em -915 mV; já para o metóxido de potássio, estão presentes dois processos de redução, o primeiro em - 1050 e o segundo em - $1215 \mathrm{mV}$, além de apresentarem correntes muito maiores, apresentando potenciais mais negativos quando se compara com os valores de potencial e corrente para a voltametria da solução aquosa de $\mathrm{KOH}$.

Em vista dos resultados observados procedeu-se à transesterificação, sendo monitorada por aproximadamente $2 \mathrm{~h}$ (50 ciclos). A Figura 4 apresenta os registros voltamétricos da emulsão de metóxido de potássio ( $\mathrm{KOH} 0,5 \mathrm{~m} / \mathrm{m}$ ), óleo e DSS, durante as $2 \mathrm{~h}$ de acompanhamento da reação. Os registros apresentados indicaram que inicialmente houve a supressão do sinal do primeiro evento de redução em -1050 mV e a definição de um único processo de redução em -1160 $\mathrm{mV}$, o qual teve o seu perfil voltamétrico definido gradualmente em função do tempo de reação.

A Figura 5 apresenta a variação da corrente de pico, obtida nos registros das voltametrias cíclicas, em função do tempo de reação de transesterificação. Os tempos usados foram obtidos através dos voltamogramas cíclicos, onde cada ciclo se completava em um tempo da ordem de 2,88 min. O aumento dos valores das correntes de pico em função do tempo sugere que há uma dependência direta do consumo de metanol com o progresso de reação, podendo ser atribuído ao fato

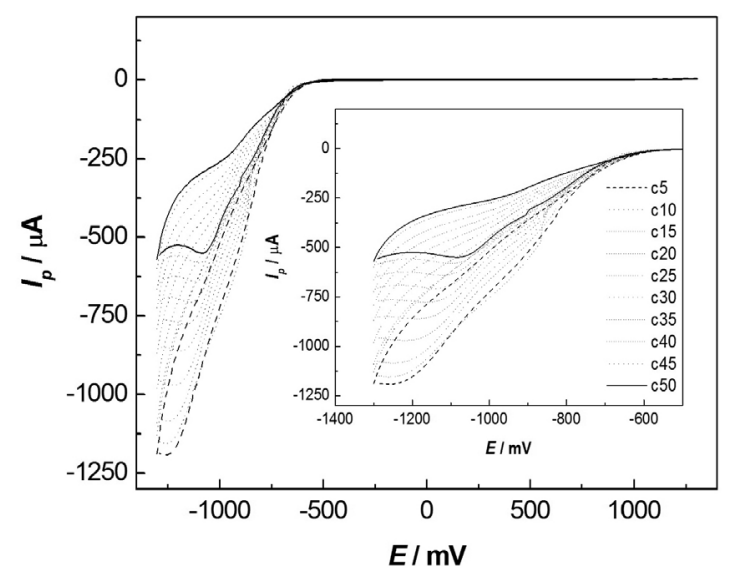

Figura 4. Registros voltamétricos do monitoramento durante $2 \mathrm{~h}$ da transesterificação. No detalhe a redução da escala do potencial para ressaltar os processos eletroquímicos

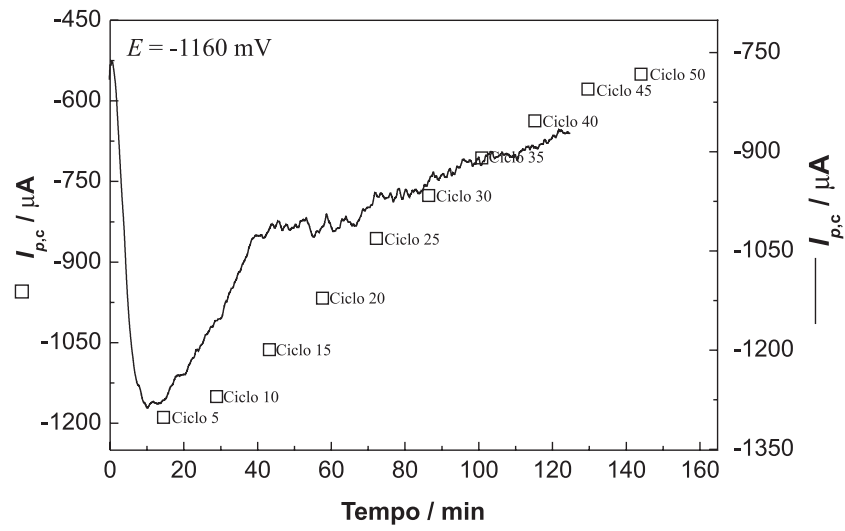

Figura 5. Variação da corrente de pico anódico em função do tempo de reação

da formação de um produto intermediário da reação de transesterificação mais eletroativo, apresentando um incremento na corrente de pico do metóxido de potássio. Porém, com o progresso da reação, o biodiesel é formado e o $\mathrm{KOH}$ regenerado, reagindo novamente com o metanol em excesso presente no meio reacional, apresentando o perfil voltamétrico semelhante ao apresentado na Figura 3. Note-se que as correntes de pico tendem a valores mais estáveis em tempos de reação acima de $2 \mathrm{~h}$, indicando que o processo de transesterificação se aproxima de seu valor máximo de conversão neste tempo de reação, como sugerido por outros autores..$^{2-4} \mathrm{~A}$ fim de confirmar se o produto final de reação correspondia realmente a biodiesel, foram analisadas uma amostra de óleo de girassol e do produto final de reação por ressonância magnética nuclear de ${ }^{1} \mathrm{H}$, como apresentado na Figura 6. No espectro do óleo, o sinal entre 4,05 e 4,35 ppm (dois duplos dubletos) é referente aos 4 átomos de hidrogênio ligados nas posições 1 e 3 do grupo glicerol do triglicerídeo. Já no espectro do produto final não há sinal referente a triglicerídeo, que corresponde assim a um éster metílico, e conclui-se que houve a conversão total do óleo em biodiesel. ${ }^{17}$

No entanto, para a confirmação desta hipótese, foram realizados experimentos para monitorar a variação da corrente de pico em função do tempo com o potencial de redução constante de $-1160 \mathrm{mV}$. A Figura 7 apresenta os resultados da variação da corrente de pico em função do tempo para três diferentes óleos - girassol, soja e canola - assim descrevendo melhor o comportamento do catalisador na reação de transesterificação. No intervalo de tempo entre 0 e 15 min houve um decaimento significativo nos valores de corrente de pico e a partir de 15 min houve o aumento gradual dos valores da corrente de pico para 


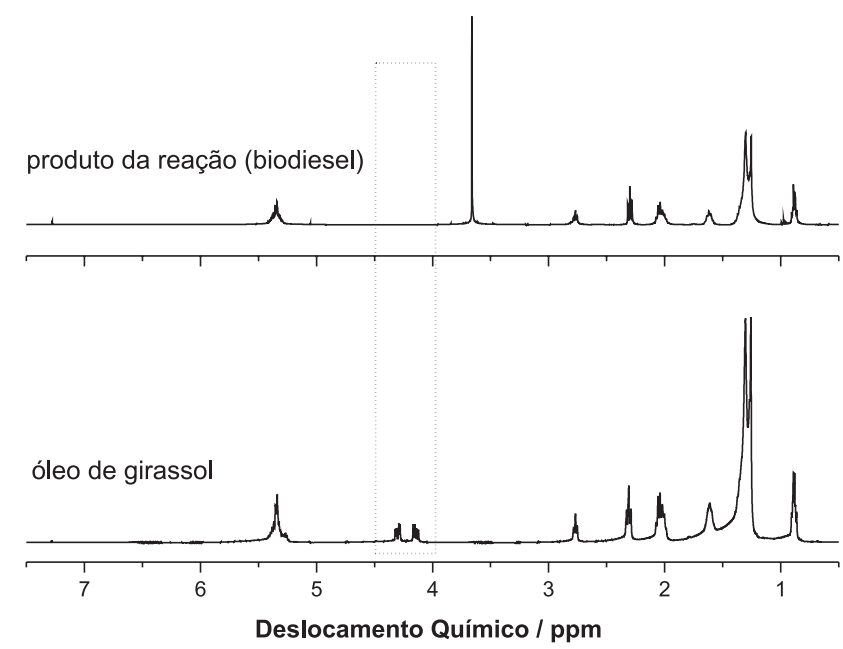

Figura 6. Espectros de ${ }^{1} H$ RMN de óleo de girassol e seu respectivo biodiesel na região entre 0,5 e 7,5 ppm. A região demarcada no centro corresponde aos deslocamentos característicos de grupos glicerol em triglicerídeos

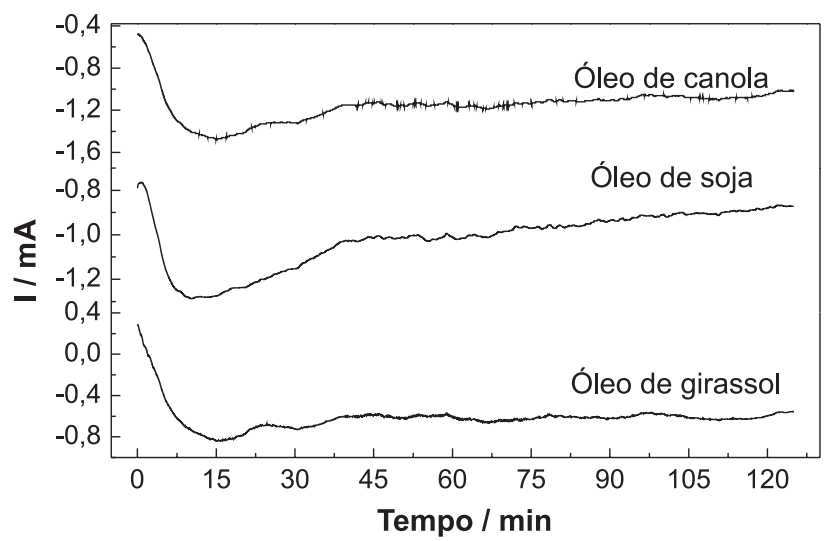

Figura 7. Registro da variação de corrente de pico em função do tempo com o potencial constante de $-1160 \mathrm{mV}$

os três casos, estabilizando os valores em até 2 h de reação. Estes resultados confirmam que os valores de corrente dependem da formação de uma espécie intermediária da reação de transesterificação e que com o progresso da reação houve o aumento da corrente, indicando a formação de metóxido de potássio no seio da solução, indicado pelo perfil voltamétrico semelhante ao apresentado anteriormente na Figura 3.

Os perfis das curvas obtidas para cada óleo são bastante semelhantes, sugerindo o mesmo comportamento eletroquímico para os óleos estudados. Além dos perfis semelhantes foi calculada a diferença de corrente entre o pico mínimo e a faixa de estabilização de corrente, obtendo-se os valores de 280, 260 e 290 A para os óleos de girassol, canola e soja, respectivamente.

Desses resultados sugeriu-se um modelo cinético para acompanhar os minutos iniciais de reação. Com o auxílio da Equação 3 foi calculada a velocidade de reação para a formação do intermediário de reação, considerando que todo o óleo usado tenha sido convertido.

$$
v=\Delta[\mathrm{A}] / \Delta \mathrm{t}
$$

A concentração inicial de óleo foi de $0,481 \mathrm{~mol} \mathrm{~L}^{-1}$, sendo consumidos 890 s para o óleo de girassol, 913 s para o óleo de canola e 600 s para o óleo de soja , como é mostrado na Figura 6. As velocidades de reação assim obtidas foram de 5,18 x 10 $0^{-4}, 5,05 \times 10^{-4}$ e $8,02 \times 10^{-4}$ $\mathrm{mol} \mathrm{s}^{-1}$ para o consumo dos óleos de girassol, canola e soja, respectivamente, o que é consistente com as condições experimentais adotadas.

\section{CONCLUSÕES}

Os resultados obtidos permitem concluir que o eletrodo de $\mathrm{TiO}_{2}$ nanoestruturado na forma de filme fino pode ser efetivo para a mensuração eletroanalítica da reação de transesterificação de óleos vegetais com metanol, catalisada por KOH. Foram identificados processos de redução frente às soluções aquosa e alcoólica, ainda indicando a formação de um intermediário de reação. Pela medida desta variação da corrente de pico foi possível observar-se um perfil de reação indicando máximo de conversão pela estabilidade da corrente de pico, valor tendendo à estabilidade acima de $2 \mathrm{~h}$ de reação. Estes resultados demonstram ser possível desenvolver uma técnica eletroanalítica rápida para acompanhamento da reação de formação de biodiesel, que deve ser melhor investigada em trabalhos futuros.

\section{AGRADECIMENTOS}

Ao CNPq, CAPES, FAPESP, FINEP e Embrapa pelo auxílio financeiro para a realização desse trabalho. Ao auxílio do Prof. Dr. G. Mambrini (UFV), que colaborou nas discussões e nas medidas experimentais de RMN para conclusão desse trabalho.

\section{REFERÊNCIAS}

1. Macedo, I. C.; Nogueira, L. A. H.; Biocombustiveis, Cadernos NAE, 2, Núcleo de Assuntos Estratégicos da Presidência da República; Secretaria de Comunicação de Governo e Gestão Estratégica: Brasília, 2005.

2. Meher, L C.; Vidya Sagar, D.; Naik, S. N.; Renewable \& Sustainable Energy Reviews 2006, 10, 248.

3. Knothe, G.; Gerpen, J. V.; Krahl, J.; Ramos, L. P.; Manual de Biodiesel, Edgarb Blucher: São Paulo, 2006.

4. Ferrari, R. A.; Oliveira, V. S.; Scabio, A.; Quim. Nova 2005, 28, 19.

5. Antunes, O. A. C.; Quim. Nova 2005, 28, 64.

6. Suarez, P. A. Z.; Meneghetti, S. M. P.; Meneghetti, M. R.; Wolf, C. R.; Quim. Nova 2007, 30, 667.

7. Watkins, R. S.; Lee, A. F.; Wilson, K.; Green Chem. 2004, 10, 335.

8. Vicente, G.; Martinez, M.; Aracil, J.; Bioresour. Technol. 2004, 92, 297.

9. Xie, W.; Li, H.; J. Am. Oil Chem. Soc. 2006, 83, 869.

10. Texter, J.; Horch, R. F.; Qutubuddin, S.; Dayalan, E.; J. Colloid Interface Sci. 1990, 135, 263.

11. Matsubara, T.; Texter, J.; J. Colloid Interface Sci. 1986, 112, 421.

12. Chunsheng, M.; Xiaoge, L.; J. Colloid Interface Sci. 2007, 312, 355.

13. Fulian, Q.; Ball, J. C.; Marken, F.; Compton, R. G.; Fisher, A. C.; Electroanalysis 2000, 12, 1012.

14. Morgenstern, M.; Cline, J.; Meyer, S.; Cataldo, S.; Energy Fuels 2006, 20, 1350 .

15. Malagutti, A. R.; Mourao, H. A. J. L.; Garbin, J. R.; Ribeiro, C.; Appl. Catal., B 2009, 90, 205.

16. Brett, C. M. A.; Brett, A. M. O.; Eletroquímica: princípios, métodos e aplicações, Oxford University Press: New York, 1996.

17. Ghesti, G. F.; Macedo, J. L.; Resck, I. S.; Dias, J. A.; Dias, S. C. L.; Energy Fuels 2007, 21, 2475. 